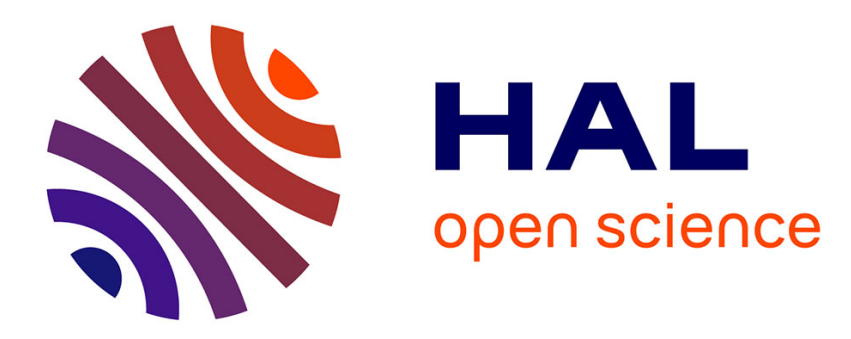

\title{
Organisation du travail, perception et gestion des risques psychosociaux
}

Marc Loriol

\section{To cite this version:}

Marc Loriol. Organisation du travail, perception et gestion des risques psychosociaux. Archives des Maladies Professionnelles et de L'Environnement, 2010, 71 (3), pp.283-286. halshs-00515931

\section{HAL Id: halshs-00515931 https://shs.hal.science/halshs-00515931}

Submitted on 8 Sep 2010

HAL is a multi-disciplinary open access archive for the deposit and dissemination of scientific research documents, whether they are published or not. The documents may come from teaching and research institutions in France or abroad, or from public or private research centers.
L'archive ouverte pluridisciplinaire HAL, est destinée au dépôt et à la diffusion de documents scientifiques de niveau recherche, publiés ou non, émanant des établissements d'enseignement et de recherche français ou étrangers, des laboratoires publics ou privés. 


\title{
Organisation du travail, perception et gestion des risques psychosociaux
}

\author{
Marc Loriol, dans : Archives des Maladies Professionnelles et de l'Environnement, \\ Volume 71, Issue 3, June 2010, Pages 283-286
}

Très fortement médiatisés, les risques psychosociaux (RPS) sont devenus un enjeu pour les pouvoirs publics qui ont sommé les partenaires sociaux et en premier lieu les entreprises de négocier et de mettre en place des stratégies de prévention. A la suite du rapport LégeronNasse, il est fréquent d'envisager la gestion du stress à travers trois grandes catégories d'action. La «prévention primaire » vise l'élimination ou le contrôle des facteurs de risque présents dans le milieu du travail en agissant sur l'organisation et les conditions de travail. La «prévention secondaire » doit aider les salariés à mieux gérer les contraintes (apprendre à faire face aux conflits, relaxation, hygiène de vie, etc.). La «prévention tertiaire », enfin correspond à la prise en charge psychologique ou médicale de ceux qui ont été mis à mal par le travail. Si l'ergonome ou le sociologue des organisations sont invités à prêter leur concours à la mise en place de la prévention primaire, c'est au médecin et surtout au psychologue qu'il reviendrait de prendre en charge les préventions secondaires et tertiaires.

Cette façon d'aborder le stress n'est adaptée ni à la complexité du monde du travail, ni à la compréhension de l'expérience des difficultés par les salariés. Faire face aux stresseurs, surmonter les contraintes du travail, est largement une affaire collective. Ensuite, l'aide psychologique est d'autant plus recherchée et bénéfique pour les salariés qu'elle s'inscrit dans un ensemble de représentations partagées et éprouvées sur la nature de l'activité, sur le rôle des psychologues dans l'institution. Enfin, les enjeux sociopolitiques et idéologiques peuvent peser sur la définition des RPS : s'agit-il de travailler en amont sur les facteurs de risque ou sur des entités cliniques encore discutées (stress, souffrance, burn out, harcèlement, etc.) ; quel terme utiliser (par exemple le stress qui renvoie plus à l'adaptation individuelle ou la souffrance qui introduit une dimension plus collective et normative) ? Du point de vue des employeurs, les problèmes de santé mentale au travail peuvent soit être niés ou renvoyés à la sphère privée (ce qui est encore très fréquent), soit pris en charge comme des questions de compétence individuelle à gérer les difficultés et de soutien psychologique. Dans le premier cas, la réponse des syndicats sera de réclamer une reconnaissance des difficultés et de la souffrance des salariés. Dans le deuxième cas, au contraire, les syndicats peuvent rejeter la notion même de stress pour mieux mettre en avant des revendications plus collectives. Mais les syndicats ne sont pas voués à rejeter le thème du stress pour contrer les tentatives d'individualisation du problème par les directions. Ils peuvent parfois chercher à imposer leur propre lecture du stress, ancrée dans l'organisation et les revendications sur les conditions de travail. Cette question d'étiquetage n'est pas anodine et dans les entreprises où les RPS sont pensés essentiellement sur le registre des manques ou des défaillances individuelles, la situation des salariés ainsi catégorisés sera plus douloureuse.

\section{Replacer la subjectivité dans un cadre collectif et organisationnel}

Dans la plupart des travaux médicaux ou psychologiques sur la perception des facteurs potentiels de stress, les représentations du travail et les réactions face aux stresseurs sont pensées comme individuelles (lieu de contrôle interne, personnalités de types A, alexithymie...). Or mes recherches, après bien d'autres, ont montré que cette médiatisation est d'abord un phénomène social, de représentations collectives forgées au sein du groupe de travail, confortées ou empêchées par l'organisation et les conditions de travail. Si certaines 
circonstances de travail sont source de souffrance dans tel service ou atelier, mais pas dans tel autre, cela ne renvoie pas à la personnalité de ceux qui y travaillent mais bien plutôt aux conditions faites à la vie collective dans le travail (faible ou fort turn over, temps pour parler du travail ou non, type de rapports hiérarchiques ou entre collègues, sociabilités collectives, mise en concurrence des salariés, etc.), conditions qui dépendent en partie des choix faits par l'employeur.

Certaines circonstances qui sont généralement tenues pour des facteurs de stress, peuvent parfois être vécues comme acceptables, voire désirables. Par exemple, sous certaines conditions, s'occuper de malades très mal en point peut être vécu comme une épreuve stimulante et valorisante par des infirmières de réanimation tandis que leurs collègues en long séjour pourront le vivre dans la souffrance. De même, pour des policiers de voie publique, arrêter un délinquant violent peut être source d'un grand prestige et de fierté professionnelle [5]. Beaucoup de situations de travail, par exemple être en contact avec des usagers ou des clients, avoir une mission floue ou mal définie, avoir des responsabilités ou des tâches variées, sont ambivalentes. Leur sens n'est a-priori ni négatif, ni positif, mais dépend de constructions locales. Par exemple, d'après l'enquête «conditions de travail» de la DARES, en 2005, $59,4 \%$ des salariés déclarent devoir « abandonner une tâche pour une autre " (ils n'étaient que $48,1 \%$ en 1991). Mais parmi ceux-ci, $16,7 \%$ déclarent qu'il s'agit d'un aspect positif de leur travail (cela peut leur permettre de casser la routine, de passer à une tâche plus valorisante, de favoriser la synthèse d'informations hétérogènes, etc.).

De façon plus ou moins prégnante suivant les groupes professionnels, chaque métier et chaque service définit un idéal, un beau travail qui sera valorisé par les pairs et la hiérarchie de proximité. Ce qui relève de ce «beau travail» mérite, plus nettement, aux yeux des salariés concernés, de faire des efforts. Les «risques du métier » sont mieux acceptés que ceux qui apparaissent comme étrangers ou sans signification pour le groupe de travail [7]. Une contrainte est d'autant mieux vécue qu'elle est perçue comme inhérente au métier, à une activité qui fait sens pour les travailleurs [4], qui est reconnue comme utile et de qualité par les collègues, la hiérarchie ou les clients.

Cela peut expliquer pourquoi, face à des conditions de travail similaires, les plaintes peuvent être plus ou moins élevées d'un lieu à un autre. Les services ou les équipes où les plaintes sont moins nombreuses sont ceux où, toutes choses égales par ailleurs, le groupe de travail parvient le mieux à donner un sens positif à des activités qui sont vécues autre part comme pénibles. Cela peut se faire par la valorisation d'une activité présentée, dans les discussions informelles entre collègues, comme faisant partie du «vrai travail ». Mais cela peut également être le cas quand une activité routinière ou pénible est transformée en une sorte de jeu ou de défi plaisant ou stimulant [2]. Dans tous les cas, cela suppose des échanges, une confiance, des valeurs partagées entre collègues de travail. Si l'employeur considère les discussions et la vie sociale au travail comme une perte de temps ou une remise en cause de son autorité et s'il tente de les réprimer ou les rend impossibles (en limitant les pauses, les moments de coprésence, les pots et autres sociabilités collectives), le travail sera vécu comme plus contraignant, plus pénible. Non seulement il aura moins de sens, mais en plus, tous les petits arrangements collectifs, l'entraide, les échanges de «ficelles du métier » ou d'encouragement, seront empêchés et ne permettront plus de faire face aux difficultés, de les gérer sans avoir recours à l'aide de la hiérarchie ou d'un psychologue extérieur.

Bien évidemment, il ne suffit pas d'avoir un idéal professionnel fort et partagé par tous pour être automatiquement en mesure de faire face aux difficultés et aux stresseurs potentiels. Si les moyens sont insuffisants ou inadaptés pour réaliser le travail selon les critères de qualité jugés satisfaisants, les salariés qui sont le plus investis et engagés dans leur travail, ceux pour qui leur mission a le plus de sens, seront justement les plus affectés. Entre 1991 et 2005, la part des salariés qui déclarent ne pas pouvoir effectuer correctement leur travail à cause d'un 
manque de temps est passée de 23 à $25 \%$; du fait d'un nombre insuffisant de collaborateurs de 27 à $30 \%$ et en raison de l'usage de logiciels informatiques inadaptés de $19 \%$ à $24 \%$ (enquête «conditions de travail » de la DARES).

La prévention secondaire, généralement pensée comme une stratégie d'amélioration des compétences individuelles (apprendre à gérer les conflits, à maîtriser ses émotions, etc.), renvoie ainsi largement à la qualité de la vie collective au travail et aux moyens laissés par l'organisation. En fait, la prévention secondaire ne devient nécessaire que lorsque l'organisation collective, la transmission des savoirs, l'entraide entre collègues, sont insuffisantes ou défaillantes, bien souvent à cause d'une organisation du travail qui refuse de reconnaître l'engagement collectif dans le travail [1]. Associer les agents à la définition des changements organisationnels permet de mieux prendre en compte les contraintes du terrain, les modes de coopération informels et les valeurs auxquelles les agents sont attachés tout en renforçant leur implication. La participation n'est toutefois pas une formule magique : elle peut déboucher sur des conflits d'intérêt ou de personnes, prendre du temps, être déstabilisante en cas de difficultés (les salariés prenant sur eux les échecs éventuels), etc. Elle fonctionnera d'autant mieux qu'elle repose sur une habitude longue de dialogue, une confiance mutuelle qui ne décrète pas mais se construit sur la durée, ce qui est difficile quand les chefs de service et les réformes se succèdent à un rythme soutenu.

\section{Un rapport au soutien psychologique socialement structuré}

De la même façon que le groupe de travail peut, dans certains cas, prendre en charge les difficultés avant qu'elles ne soient productrices de stress ou de souffrance (grâce à un changement informel dans la répartition des tâches ou une nouvelle définition des missions peuvent, par exemple), les collègues peuvent, quand la confiance et la solidarité sont suffisantes, offrir un soutien psychologique et du réconfort à ceux qui se sentent mal dans leur peau. Une telle situation a été observée, par exemple, à l'occasion d'une recherche sur le stress dans la police [5], dans des brigades de nuit rassemblant des anciens travaillant de longue date ensemble. Dans ce cas, les salariés ont alors tendance à préférer une aide interne, par les pairs, à une aide extérieure par un professionnel (psychologue psychanalyste ou psychiatre), perçu comme étranger au groupe et ne connaissant pas le métier et ses difficultés. Parler de ses problèmes, de ses émotions, d'éventuelles fragilités à un psychologue ne va pas de soi, notamment pour des salariés ayant un faible niveau d'étude ou dont le travail ne favorise pas une lecture psychologique du monde. C'est pourquoi, dans mes recherches, j'ai pu également constater une certaine réticence au soutien psychologique de la part de médecins hospitaliers ou de commissaires de police. A l'inverse, les infirmières, parce qu'elles valorisent dans leur travail la dimension psychique de la prise en charge des patients, notamment pour se distinguer des médecins, plus centrés sur la prise en charge des troubles somatiques, ont un a-priori plus favorable à l'égard de la prise en charge psychologique. Or les catégories professionnelles réticentes vis-à-vis du savoir psychologique et des méthodes d'accompagnement sont moins réceptives, tirent moins profit du soutien.

Ceci-dit, il n'y a pas de déterminisme strict et certaines catégories de salariés, a-priori peu enclines à aborder leurs difficultés sous l'angle psychologique et émotionnel, peuvent néanmoins accepter une aide si celle-ci n'est pas vécue comme stigmatisante ou infantilisante. Les machinistes receveurs de la RATP se sont d'abord vus, dans les années 1990, proposer un soutien psychologique fondé sur une démarche cognitivo-comportementale (apprendre à gérer ses émotions et l'agressivité des passagers) dans laquelle ils ne se sont pas reconnus. Les syndicats et des psychologues internes à la Régie ont alors développé une approche différente, plus fondée sur la connaissance des métiers, de leur culture, et doublée d'une vigilance accrue sur les causes organisationnelles du stress et des agressions. Aller voir le psychologue n'est 
alors plus perçu comme un signe d'insuffisance ou de fragilité personnelle et n'empêche pas de revendiquer une amélioration des conditions de travail ou la mise en place de démarches de prévention en amont.

La prévention tertiaire est qualifiée de «prévention » dans la mesure où elle a pour objectif d'éviter que les traumatismes psychologiques ne débouchent sur des troubles plus graves (dépressions, suicides...). Mais l'efficacité de cet accompagnement est moins liée au fait de verbaliser ses émotions pour «vider son sac », qu'à la possibilité de donner un sens acceptable et partagé aux traumatismes subis dans le travail [3,7]. C'est pourquoi, une fois encore, dans les entreprises ou les services dans lesquels le sens du travail est évident pour chacun, le besoin d'un soutien psychologique est moins fort car les salariés font face plus collectivement aux difficultés qui ne sont plus vécues comme des mises en causes personnelles.

Si un accompagnement psychologique est nécessaire, il ne faut pas que celui qui en bénéficie se sente culpabilisé. Dans mes recherches, j'ai recueilli les témoignages de salariés qui avait eu le sentiment que le psychologue qu'ils consultaient partait du postulat de départ selon lequel la cause de leur problème était à chercher en eux-mêmes (dans leur enfance, leur rapport à la violence, leurs motivations inconscientes, etc.) et non dans leur environnement de travail. Pour ne pas faire des personnes suivies des «malades », coupés de l'entreprise et dont l'identité ne serait plus définie que par le stress ou la souffrance, il est au contraire indispensable que le suivi soit organisé par des professionnels (psychologues ou médecins du travail) qui connaissent bien le milieu professionnel concerné, ses peines et ses joies. Ainsi, ils pourront rattacher la souffrance vécue par le salarié aux difficultés et problèmes du métier ou de l'entreprise et aider la personne à prendre du recul. A l'inverse, des psychologues qui par ignorance du milieu de travail ou par peur de mécontenter les directions ne font qu'appliquer des recettes standard, qu'elles soient d'inspiration psychanalytique (par exemple chercher dans le passé de la personne l'origine de sa réaction) ou cognitivo-comportementale (voir le verre à moitié plein plutôt que le verre à moitié vide), renvoient l'individu à lui-même. La place des psychologues dans l'institution est donc importante. Des psychologues en situation de précarité, qui craignent que leur contrat ne soit pas renouvelé, qui interviennent dans un grand nombre de secteur sans pouvoir rester suffisamment dans chacun d'entre eux, sont démunis dés lors qu'il s'agit de comprendre finement les enjeux locaux de l'engagement dans le travail, les conditions sociales d'une activité qui pourra être source de fierté professionnelle.

A ce titre, les acteurs les mieux à même d'assurer la prévention - à la fois primaire, secondaire et tertiaire - des risques psychosociaux restent encore ceux qui sont au cœur de la vie de l'entreprise : l'encadrement, le CHSCT, la médecine du travail; à condition qu'ils soient suffisamment sensibilisés à l'importance de la vie collective au travail. Le médecin du travail notamment doit pouvoir jouer un rôle de coordination des différents intervenants en prévention des risques professionnels. Concernant les RPS il semble indispensable d'assurer des échanges d'expertise interdisciplinaires (médecine du travail, épidémiologie, ergonomie, sociologie et psychologie) et de poser des gardes-fous sur l'application de recettes trop standardisées issues d'une seule discipline. Quand des dispositifs de prévention des RPS sont mis en place sans participation active de la médecine du travail, le risque est plus élevé d'avoir une démarche essentiellement tournée vers l'écoute psychologique, sans ancrage dans le collectif du travail ni l'activité concrète.

\section{Références :}

Alter N., Donner et prendre : la coopération en entreprise, La Découverte, 2009. 
2 Cartron, D. « Le sociologue pris en sandwich! Retour sur une observation participante dans un fast-food », Travail et Emploi, n 94, Avril 2003 : 59-64.

3 Fassin D., Rechtman R., L'Empire du traumatisme. Enquête sur la condition de victime, Paris, Flammarion, 2007.

$4 \quad$ Hanique F. Le sens du travail. Chronique de la modernisation au guichet, ERES, Col «Sociologie clinique », 2004.

5 Loriol M., Boussard V., Caroly S., «La résistance à la psychologisation des difficultés au travail. Le cas des policiers de voie publique », Actes de la recherche en sciences sociales, $\mathrm{n}^{\circ}$ 166, 2006 : 92-101.

6 Rimé B., Le partage social des émotions, PUF, 2005.

$7 \quad$ Roche, P. Une santé d'acier, Editions du CNRS, 1987. 